\title{
A constitutive analysis of transient and steady-state elongational viscosities of bidisperse polystyrene blends
}

Wagner, Manfred H.; Rolon-Garrido, Victor H.; Nielsen, Jens Kromann; Rasmussen, Henrik K.; Hassager, Ole

Published in:

Journal of Rheology

Link to article, DOI:

$10.1122 / 1.2807442$

Publication date:

2008

Document Version

Publisher's PDF, also known as Version of record

Link back to DTU Orbit

Citation (APA):

Wagner, M. H., Rolon-Garrido, V. H., Nielsen, J. K., Rasmussen, H. K., \& Hassager, O. (2008). A constitutive analysis of transient and steady-state elongational viscosities of bidisperse polystyrene blends. Journal of Rheology, 52(1), 67-86. https://doi.org/10.1122/1.2807442

\section{General rights}

Copyright and moral rights for the publications made accessible in the public portal are retained by the authors and/or other copyright owners and it is a condition of accessing publications that users recognise and abide by the legal requirements associated with these rights.

- Users may download and print one copy of any publication from the public portal for the purpose of private study or research.

- You may not further distribute the material or use it for any profit-making activity or commercial gain

- You may freely distribute the URL identifying the publication in the public portal 


\title{
A constitutive analysis of transient and steady-state elongational viscosities of bidisperse polystyrene blends
}

\author{
Manfred H. Wagner ${ }^{\mathrm{a})}$ and Victor H. Rolón-Garrido \\ Polymertechnik / Polymerphysik, Technical University of Berlin, \\ Fasanenstrasse 90, D-10623 Berlin, Germany \\ Jens Kromann Nielsen
}

The Danish Polymer Center, Department of Chemical Engineering, Technical University of Denmark, DK-2800 Kgs. Lyngby, Denmark

Henrik K. Rasmussen

The Danish Polymer Center, Department of Manufacturing Engineering and Management, Technical University of Denmark, DK-2800 Kgs. Lyngby, Denmark

\section{Ole Hassager}

The Danish Polymer Center, Department of Chemical Engineering, Technical University of Denmark, DK-2800 Kgs. Lyngby, Denmark

(Received 22 October 2006; final revision received 26 September 2007)

\section{Synopsis}

The transient and steady-state elongational viscosity data of three bidisperse polystyrene blends were investigated recently by Nielsen et al. [J. Rheol. 50, 453-476 (2006)]. The blends contain a monodisperse high molar mass component $\left(M_{L}=390 \mathrm{~kg} / \mathrm{mol}\right)$ in a matrix of a monodisperse small molar mass component (either $M_{S}=103 \mathrm{~kg} / \mathrm{mol}$ or $M_{S}=52 \mathrm{~kg} / \mathrm{mol}$ at two different weight fractions). The experimental data are analyzed in the framework of the molecular stress function model of Wagner et al. [J. Rheol. 49, 1317-1327 (2005)], which is based on the assumption of a strain-dependent tube diameter and the interchain pressure term of Marrucci and Ianniruberto [Macromolecules 37, 3934-3942 (2004)]. The dilution of the long chains in the matrix of the short chains is identified as the origin of a drastic increase in the tube-diameter relaxation time of the long chains, leading to a large stretching potential of the long-chain component and an increasing steady-state elongational viscosity with increasing strain rate. In addition, in the dilution regime, a transition from affine chain stretch to nonaffine tube squeeze with decreasing strain rate is identified. The dilution regime ends at a critical strain rate, when the tube diameter of the supertubes created by the interaction of the long chains among themselves, is reduced by deformation to the tube diameter of the bulk. A nonlinear extension of the basic double reptation concept is developed comprising all of these different phenomena, and allowing (albeit by use of

\footnotetext{
a) Author to whom correspondence should be addressed; electronic mail: manfred.wagner@tu-berlin.de
} 
empirical linear-viscoelastic shift factors to correct the linear-viscoelastic predictions) for a quantitative description of the transient and steady-state elongational viscosities of the bidisperse polystyrene blends. (C) 2008 The Society of Rheology. [DOI: 10.1122/1.2807442]

\section{INTRODUCTION}

Based on the reptation theory first proposed by de Gennes (1971), Doi and Edwards (DE) developed the concept of the tube model [Doi and Edwards (1978)], in which a polymer chain is enclosed in a tubular region delimited by the neighboring macromolecules. The DE model is able to describe some rheological phenomena like the damping function in step-shear strain [e.g., Isono et al. (1991)] as well as constant strain-rate uniaxial and biaxial [e.g., Urakawa et al. (1995)] deformation experiments for narrow distribution polymers in limited deformation rate regimes, but its general performance can be rated as more qualitative than quantitative [Larson (1988)]. Even for monodisperse polymer melts, contour-length fluctuations [Doi (1981, 1983); Ketzmerick and Öttinger (1989); O’Connor and Ball (1992); Milner and McLeish (1998)] have to be invoked to get agreement with the experimentally observed power law index of 3.4 [Berry and Fox (1968)] between the zero shear viscosity $\eta_{0}$ and molar mass $M$, while for polydisperse systems concepts of double reptation [Tsenoglou (1987); des Cloizeaux (1988, 1990); Wasserman and Graessley (1992)] or tube dilation [Milner and McLeish (1998)] have to be considered to get quantitative agreement between linear-viscoelastic data and modeling [e.g., van Ruymbeke et al. (2002)].

In the nonlinear viscoelastic regime, an identified shortcoming of the original DE theory is the lack of predictive power in general extensional flows [Wagner (1990); Wagner et al. (2005a)], since the stress is assumed to originate by chain orientation only [Doi and Edwards $(1978,1979)]$, thereby ignoring the possibility of chain stretching, which is especially important in extensional deformations. Theories accounting for chain stretch have been proposed either based on the general idea that chain stretching starts to be significant at Deborah numbers $D e=\dot{\varepsilon} \tau_{R}>1$ (where $\dot{\varepsilon}$ is the strain rate and $\tau_{R}$ the Rouse time of the chain) [e.g., Doi and Edwards (1986); Marrucci and Grizzuti (1988); Mead and Leal (1995); Hua and Schieber (1998); Öttinger (1999)], or based on the assumption that chain stretch is a consequence of tube contraction by deformation [Marrucci and Hermans (1980); Wagner and Schaeffer (1992)].

A consequence of ignoring chain stretch is that the DE theory [Doi and Edwards (1979)] predicts a steady-state elongational viscosity $\eta_{u s}$ scaling:

$$
\eta_{u s} \propto \dot{\varepsilon}^{\phi}
$$

with an exponent $\phi=-1$. However, recent elongational viscosity measurements of nearly monodisperse polystyrene melts have shown a power-law scaling of the steady-state elongational viscosity with approximately $\phi=-1 / 2$ [Bach et al. (2003a)]. The exponent $\phi=-1 / 2$ was explained by Marrucci and Ianniruberto (2004) on the basis of scalar scaling arguments considering tube contraction due to the applied deformation, which is balanced by an internal chain pressure against the tube wall.

Introducing the interchain pressure term of Marrucci and Ianniruberto (2004) into the molecular stress function (MSF) model [Wagner et al. (2001)], the steady-state as well as the transient elongational viscosities of four nearly monodisperse polystyrene melts could be modeled quantitatively by use of a single nonlinear material parameter, the tube diameter relaxation time $\tau_{a}$ [Wagner et al. (2005a)]. It is worth mentioning that for the 
highest molar mass polystyrene PS390K, a scaling of the steady-state elongational viscosity close to $\eta_{u s} \propto \dot{\varepsilon}^{-0.4}$ was predicted, which is in quantitative agreement with the experiment.

The experimental results of Bach et al. (2003a) were confirmed by Luap et al. (2005), who simultaneously measured stress and birefringence during elongation of two polystyrene melts with narrow molar mass distribution. The samples did not follow the stress optical rule. Elongational viscosity data could again be modeled quantitatively by the MSF model of Wagner et al. (2005a), which was modified to account for non-Gaussian chain extension [Rolón-Garrido et al. (2006)]. The tube diameter relaxation times were found to scale with $\mathrm{M}^{2}$. While the transient elongational viscosity shows a small dependence on finite extensibility, the predicted steady-state elongational viscosity is not affected by non-Gaussian effects.

A further challenge is the quantitative constitutive modeling of polydisperse samples, which are those of industrial interest. A first step in this direction has been the study of bidisperse samples where one component has a higher molar mass than the other one [Masuda et al. (1970); Watanabe et al. (1985); Ylitalo et al. (1991)]. It has been elucidated that in this case the concept of reptation is not sufficient to describe the linear viscoelasticity of the samples, since the long chains move in an environment of relaxed constraints due to the faster reptation of the short chains, or in other words, like in an ordinary solvent [Daoud and de Gennes (1979)]. The majority of the studies have been focused on the linear-viscoelastic modeling of bidisperse blends [Marrucci (1985); Frischknecht and Milner (2002); Wang et al. (2003)], which has lead to the development of concepts like tube dilation [Doi et al. (1987); Milner and McLeish (1998)] or double reptation [Tsenoglou (1987); des Cloizeaux (1988, 1990); Wasserman and Graessley (1992)].

The nonlinear viscoelastic regime of bidisperse systems has barely been studied in either solution [Gupta et al. (2000); Ye et al. (2003)] or in the melt state. Recently, Nielsen et al. (2006) reported experimental data of bidisperse polystyrene blends measured in uniaxial elongation in a broad range of elongational rates. These data are especially interesting, since the deformations achieved were large enough to determine the steady-state elongational viscosities.

The objective of the present contribution is to quantitatively analyze the transient and steady-state elongational viscosities of the bidisperse polystyrene melts investigated by Nielsen et al. (2006) on the basis of the MSF model, and identify the basic physical phenomena that govern the rheology of bidisperse blends.

\section{EXPERIMENT}

The experimental data discussed are those of Nielsen et al. (2006). Three different polystyrene blends were investigated whose blend components are three polystyrenes with mass-average molar masses of $52 \mathrm{~kg} \mathrm{~mol}^{-1}$ (PS50k), $103 \mathrm{~kg} \mathrm{~mol}^{-1}$ (PS100k), and $390 \mathrm{~kg} \mathrm{~mol}^{-1}$ (PS390k), and polydispersities of 1.026, 1.022, and 1.060, respectively. Table I summarizes the composition of the blends. A constitutive analysis of the elongational viscosities of the nearly monodisperse blend components has already been reported elsewhere [Wagner et al. (2005a)]. The molecular and linear-viscoelastic characterization of the blend components are summarized in Table II.

The linear-viscoelastic experiments performed at $150{ }^{\circ} \mathrm{C}$ were shifted to $130{ }^{\circ} \mathrm{C}$ using standard time-temperature shifting according to the WLF equation with $\mathrm{c}_{1}=8.86$ and $\mathrm{c}_{2}$ $=101.6{ }^{\circ} \mathrm{C}$ at $\mathrm{T}_{0}=136.5^{\circ} \mathrm{C}$, according to Bach et al. (2003a). From $\mathrm{G}^{\prime}$ and $\mathrm{G}^{\prime \prime}$ data of the blends, we derived the discrete relaxation spectra with partial moduli $g_{i}$ and relaxation 
TABLE I. Composition and characterization of bidisperse blends (relaxation spectra at $T=130{ }^{\circ} \mathrm{C}$ ).

\begin{tabular}{|c|c|c|c|c|c|c|}
\hline & \multicolumn{2}{|c|}{ Blend 1} & \multicolumn{2}{|c|}{ Blend 2} & \multicolumn{2}{|c|}{ Blend 3} \\
\hline $\begin{array}{l}\text { PS50K } \\
\text { w/w\% }\end{array}$ & \multicolumn{2}{|c|}{95.98} & \multicolumn{2}{|c|}{85.63} & \multicolumn{2}{|c|}{0} \\
\hline $\begin{array}{l}\text { PS100K } \\
\text { w/w } \%\end{array}$ & \multicolumn{2}{|c|}{0} & \multicolumn{2}{|c|}{0} & \multicolumn{2}{|c|}{85.98} \\
\hline $\begin{array}{l}\text { PS390K } \\
\text { w/w\% }\end{array}$ & \multicolumn{2}{|c|}{4.02} & \multicolumn{2}{|c|}{14.37} & \multicolumn{2}{|c|}{14.02} \\
\hline$G_{r}[-]$ & \multicolumn{2}{|c|}{0.499} & \multicolumn{2}{|c|}{0.499} & \multicolumn{2}{|c|}{0.064} \\
\hline$\eta_{0}[$ Pa.s $]$ & \multicolumn{2}{|c|}{$1.49 \times 10^{6}$} & \multicolumn{2}{|c|}{$5.91 \times 10^{6}$} & \multicolumn{2}{|c|}{$1.46 \times 10^{7}$} \\
\hline$J_{e}^{0}\left[\mathrm{~Pa}^{-1}\right]$ & \multicolumn{2}{|c|}{$4.53 \times 10^{-3}$} & \multicolumn{2}{|c|}{$7.57 \times 10^{-4}$} & \multicolumn{2}{|c|}{$1.81 \times 10^{-4}$} \\
\hline$\tau_{w}[s]$ & \multicolumn{2}{|c|}{$6.75 \times 10^{3}$} & \multicolumn{2}{|c|}{$4.48 \times 10^{3}$} & \multicolumn{2}{|c|}{$2.63 \times 10^{3}$} \\
\hline \multirow[t]{10}{*}{$\tau_{D}[s]$} & \multicolumn{2}{|c|}{$9.05 \times 10^{5}$} & \multicolumn{2}{|c|}{$2.75 \times 10^{5}$} & \multicolumn{2}{|c|}{$7.44 \times 10^{4}$} \\
\hline & $g_{i}[\mathrm{~Pa}]$ & $\lambda_{i}[\mathrm{~s}]$ & $g_{i}[\mathrm{~Pa}]$ & $\lambda_{i}[\mathrm{~s}]$ & $g_{i}[\mathrm{~Pa}]$ & $\lambda_{i}[\mathrm{~s}]$ \\
\hline & $5.705 \times 10^{5}$ & $1.111 \times 10^{-1}$ & $6.187 \times 10^{5}$ & $9.688 \times 10^{-2}$ & $3.296 \times 10^{5}$ & $2.383 \times 10^{-1}$ \\
\hline & $1.023 \times 10^{5}$ & $1.459 \times 10^{0}$ & $9.702 \times 10^{4}$ & $1.134 \times 10^{0}$ & $6.568 \times 10^{4}$ & $2.742 \times 10^{0}$ \\
\hline & $8.248 \times 10^{4}$ & $6.163 \times 10^{0}$ & $9.919 \times 10^{4}$ & $5.859 \times 10^{0}$ & $5.957 \times 10^{4}$ & $1.502 \times 10^{1}$ \\
\hline & $1.418 \times 10^{3}$ & $9.399 \times 10^{1}$ & $9.908 \times 10^{3}$ & $2.762 \times 10^{1}$ & $6.675 \times 10^{4}$ & $6.200 \times 10^{1}$ \\
\hline & $4.409 \times 10^{2}$ & $7.914 \times 10^{2}$ & $3.126 \times 10^{3}$ & $1.569 \times 10^{2}$ & $5.086 \times 10^{3}$ & $3.978 \times 10^{2}$ \\
\hline & $3.460 \times 10^{1}$ & $5.207 \times 10^{3}$ & $1.550 \times 10^{3}$ & $7.731 \times 10^{2}$ & $2.166 \times 10^{3}$ & $2.731 \times 10^{3}$ \\
\hline & $1.291 \times 10^{0}$ & $8.266 \times 10^{4}$ & $1.116 \times 10^{3}$ & $2.395 \times 10^{3}$ & $8.535 \times 10^{1}$ & $1.575 \times 10^{4}$ \\
\hline & & & $1.452 \times 10^{1}$ & $3.622 \times 10^{4}$ & & \\
\hline
\end{tabular}

times $\lambda_{i}$ by use of the IRIS program [Winter and Mours (2003)], which are also presented in Table I. Elongational viscosities of the polystyrene blends were measured for elongational rates between 0.00015 and $0.3 \mathrm{~s}^{-1}$ by using a filament stretching rheometer [Bach et al. (2003b)].

\section{THEORY}

The general structure of the MSF model [Wagner and Schaeffer (1992); Wagner et al. (2001, 2003)] is as follows: The tube diameter $a$ of a tube segment is assumed to be independent of the orientation of the tube segment, and to decrease from its equilibrium value $a_{0}$ to a value $a$ with increasing deformation.

TABLE II. Characterization of blends components (Relaxation spectra at $T=130{ }^{\circ} \mathrm{C}$ ) [Wagner et al. (2005a)].

\begin{tabular}{|c|c|c|c|c|c|c|}
\hline & \multicolumn{2}{|c|}{ PS50K } & \multicolumn{2}{|c|}{ PS100K } & \multicolumn{2}{|c|}{ PS390K } \\
\hline$\eta_{0}[$ Pa.s $]$ & \multicolumn{2}{|c|}{$8.65 \times 10^{5}$} & \multicolumn{2}{|c|}{$8.13 \times 10^{6}$} & \multicolumn{2}{|c|}{$7.57 \times 10^{8}$} \\
\hline$J_{e}^{0}\left[\mathrm{~Pa}^{-1}\right]$ & \multicolumn{2}{|c|}{$5.81 \times 10^{-6}$} & \multicolumn{2}{|c|}{$1.15 \times 10^{-5}$} & \multicolumn{2}{|c|}{$1.50 \times 10^{-5}$} \\
\hline$\tau_{R}[s]$ & \multicolumn{2}{|c|}{6.4} & \multicolumn{2}{|c|}{22.9} & \multicolumn{2}{|c|}{329} \\
\hline$\tau_{w}[s]$ & \multicolumn{2}{|c|}{5.0} & \multicolumn{2}{|c|}{94} & \multicolumn{2}{|c|}{11351} \\
\hline \multirow[t]{10}{*}{$\tau_{a}[s]$} & \multicolumn{2}{|c|}{141} & \multicolumn{2}{|c|}{203} & \multicolumn{2}{|c|}{1462} \\
\hline & $g_{i}[\mathrm{~Pa}]$ & $\lambda_{i}[\mathrm{~s}]$ & $g_{i}[\mathrm{~Pa}]$ & $\lambda_{i}[\mathrm{~s}]$ & $g_{i}[\mathrm{~Pa}]$ & $\lambda_{i}[\mathrm{~s}]$ \\
\hline & $9.15 \times 10^{6}$ & $2.91 \times 10^{-3}$ & $4.10 \times 10^{5}$ & $6.73 \times 10^{-2}$ & $4.63 \times 10^{5}$ & $1.04 \times 10^{-1}$ \\
\hline & $3.71 \times 10^{5}$ & $9.61 \times 10^{-2}$ & $1.02 \times 10^{7}$ & $3.06 \times 10^{-3}$ & $7.79 \times 10^{4}$ & $1.07 \times 10^{0}$ \\
\hline & $1.40 \times 10^{5}$ & $9.85 \times 10^{-1}$ & $1.47 \times 10^{5}$ & $4.77 \times 10^{-1}$ & $3.00 \times 10^{4}$ & $9.70 \times 10^{0}$ \\
\hline & $1.05 \times 10^{5}$ & $6.33 \times 10^{0}$ & $7.73 \times 10^{4}$ & $3.40 \times 10^{0}$ & $2.55 \times 10^{4}$ & $5.16 \times 10^{1}$ \\
\hline & & & $6.77 \times 10^{4}$ & $1.93 \times 10^{1}$ & $3.33 \times 10^{4}$ & $2.35 \times 10^{2}$ \\
\hline & & & $7.39 \times 10^{4}$ & $8.51 \times 10^{1}$ & $4.31 \times 10^{4}$ & $1.14 \times 10^{3}$ \\
\hline & & & $1.11 \times 10^{2}$ & $1.35 \times 10^{3}$ & $5.15 \times 10^{4}$ & $5.97 \times 10^{3}$ \\
\hline & & & & & $2.28 \times 10^{4}$ & $1.71 \times 10^{4}$ \\
\hline
\end{tabular}


The extra stress tensor $\boldsymbol{\sigma}(t)$ of the MSF model is then given by a history integral of the form

$$
\boldsymbol{\sigma}(t)=\int_{-\infty}^{t} m\left(t-t^{\prime}\right) f^{2} S_{D E}^{I A}\left(t, t^{\prime}\right) d t^{\prime},
$$

where $m\left(t-t^{\prime}\right)$ is the linear-viscoelastic memory function describing the reptation kinetics of tube segments. $m\left(t-t^{\prime}\right)$ will be represented in the following by a sum of the discrete relaxation modes,

$$
m\left(t-t^{\prime}\right)=\sum_{i=1}^{N}\left(\frac{g_{i}}{\lambda_{i}}\right) e^{-\left(t-t^{\prime}\right) / \lambda_{i}} .
$$

The molecular stress function $f=f\left(t, t^{\prime}\right)$ is the inverse of the relative tube diameter,

$$
f\left(t, t^{\prime}\right)=\frac{a_{0}}{a\left(t, t^{\prime}\right)},
$$

representing the relative tension or stretch of tube segments, with $t^{\prime}$ indicating the time of creation of a tube segment due to the reptation of the macromolecule.

The relative strain measure $S_{D E}^{I A}\left(t, t^{\prime}\right)$ represents the orientational contribution to the extra stress tensor originating from an affine rotation of the tube segments assuming "independent alignment (IA)" [Doi and Edwards (1978)], and is given by

$$
S_{D E}^{I A}\left(t, t^{\prime}\right) \equiv 5\left\langle\frac{u^{\prime} u^{\prime}}{u^{\prime 2}}\right\rangle_{\mathrm{o}}=5 S\left(t, t^{\prime}\right),
$$

with $\boldsymbol{S}=\boldsymbol{S}\left(t, t^{\prime}\right)$ being the relative second order orientation tensor. $\boldsymbol{u}^{\prime} \boldsymbol{u}^{\prime}$ is the dyad of a deformed unit vector $\boldsymbol{u}^{\prime}=\boldsymbol{u}^{\prime}\left(t, t^{\prime}\right)$,

$$
\boldsymbol{u}^{\prime}=\boldsymbol{F}_{t}^{-1} \cdot \boldsymbol{u}
$$

$\boldsymbol{F}_{t}^{-1}=\boldsymbol{F}_{t}^{-1}\left(t, t^{\prime}\right)$ is the relative deformation gradient tensor, and $u^{\prime}$ is the length of $\boldsymbol{u}^{\prime}$. The orientation average is indicated by $\langle\cdots\rangle_{0}$,

$$
\langle\cdots\rangle_{\mathrm{o}} \equiv \frac{1}{4 \pi} \oiint[\cdots] \sin \theta_{o} d \theta_{o} d \varphi_{o}
$$

i.e., an average over an isotropic distribution of unit vectors $\boldsymbol{u}$.

The square of the relative stretch of the chain segments, $f^{2}$, is related to the strain energy stored in the polymeric system. It has been determined as solution of evolution equations derived from energy balance [Wagner et al. (2001)] or force balance arguments [Wagner et al. (2005a)], depending on the molecular architecture of the polymer melts considered and the reaction of the polymer chains to the macroscopic deformation imposed. Two limiting cases are discussed below.

\section{A. Chain stretch by "tube squeeze"}

According to the tube model [Doi and Edwards (1978)], the tube represents the effect of the mean field of the surrounding macromolecules. Wagner et al. (2001) assumed an affine deformation of the average relative tube cross-sectional area $A\left(t, t^{\prime}\right)=1 / f^{2}$ to describe the chain stretch of polydisperse linear polymer melts, which can be expressed as 


$$
\frac{\partial A}{\partial t}=-(\boldsymbol{\kappa}: S) A
$$

from which follows immediately:

$$
\frac{\partial f^{2}}{\partial t}=f^{2}(\boldsymbol{\kappa}: S),
$$

with the initial condition $f\left(t=t^{\prime}, t^{\prime}\right)=1$. $\boldsymbol{\kappa}$ is the velocity gradient tensor. Equation (9) has the analytical solution

$$
f^{2}=e^{\left\langle\ln \left(u^{\prime}\right)\right\rangle},
$$

i.e., $f^{2}$ is an exponential of the orientational free energy $3 k_{b} T\left\langle\ln u^{\prime}\right\rangle_{0}$, with $k_{B}$ being the Boltzmann constant and $T$ the absolute temperature. Since Eq. (10) implies that $f^{2}$ (rather than $f$ ) is proportional to the average deformation $e^{\left\langle\ln \left(u^{\prime}\right)\right\rangle}$, Eq. (10) was sometimes called the linear molecular stress function theory. It should be noted that while the tube cross sectional area is deformed affinely, the physical length of the tube is not. Therefore, Eq. (10) implies a nonaffine deformation [Wagner et al. (2000)].

This approach was used successfully to predict the transient viscosity up to steady state in uniaxial, equibiaxial, and planar extension of polydisperse linear polymer melts [Wagner et al. (2001, 2005b)]. Regarding the hypothesis leading to Eq. (8), it may be stated that stretch of the test chain is caused by the "squeeze" of the surrounding polymer chains. We call this "stretch by tube squeeze" in the following.

Equations (8) and (9) consider only the hyperelastic limit. Extending these equations to include convective constraint release allows one to describe the steady-state viscosities as reported elsewhere [Wagner et al. (2001)].

\section{B. Affine chain stretch}

In contrast to polydisperse linear polymer melts, modeling the strain hardening of linear monodisperse polymer melts was only possible by assuming an (on average) affine tube deformation. In terms of the tube diameter $a\left(t, t^{\prime}\right)$, this means that $a$ changes with deformation from its equilibrium value $a_{0}$ according to

$$
\frac{\partial a}{\partial t}=-(\boldsymbol{\kappa}: S) a
$$

With $\partial a / \partial t=-a_{0}\left(1 / f^{2}\right) \partial f / \partial t$ follows the evolution equation for $f^{2}$ as:

$$
\frac{\partial f^{2}}{\partial t}=2 f^{2}(\boldsymbol{\kappa}: S)
$$

Equation (12) has the analytical solution

$$
f^{2}=e^{2\left\langle\ln \left(u^{\prime}\right)\right\rangle},
$$

which was sometimes called the quadratic molecular stress function Theory [Wagner et al. (2000)]. Obviously, in this case tube stretch is caused by an (on average) affine deformation of the tube formed by the surrounding polymer chains. We call this "affine chain stretch" in the following. The assumption of affine chain stretch, although unbound, has been shown to model quantitatively the slope of the transient elongational viscosities (before reaching the steady state) of nearly monodisperse polystyrenes [Wagner et al. (2005a)]. 
At higher strains, chain stretching is balanced by the interchain pressure term of Marrucci and Ianniruberto (2004), which results in an evolution equation:

$$
\frac{\partial f^{2}}{\partial t}=2 f^{2}\left[(\boldsymbol{\kappa}: S)-\frac{1}{\tau_{a}} f\left(f^{3}-1\right)\right],
$$

leading to steady-state viscosities. The first term on the right hand side describes affine chain stretch as discussed previously, and the second term represents the interchain pressure contribution. $\tau_{a}$ has been called the tube-diameter relaxation time, representing the relaxation of the topological constraints caused by the many surrounding chains [Wagner et al. (2005a)]. Equation (14) has successfully been used to model the transient and steady-state elongational viscosities of nearly monodisperse polystyrene melts of Bach et al. (2003a) and Luap et al. (2005) [Wagner et al. (2005a); Rolón-Garrido et al. (2006)], and is the starting point of the following analysis.

\section{ANALYSIS OF EXPERIMENTAL ELONGATIONAL VISCOSTY DATA OF BIDISPERSE POLYSTYRENE BLENDS}

In a bidisperse system containing long (L) and short (S) molecules with $M_{L} \gg M_{S}$, reptation of the short molecules is much faster than reptation of the long chains. In the case of elongation at a Deborah number $D e_{S}=\dot{\varepsilon} \tau_{w, S}<1$, where $\tau_{w, S}$ is the longest relaxation time of the short chains, the short molecules are excited in the linear-viscoelastic regime, while all strain hardening originates from the nonlinear viscoelasticity of the long chains only. Effectively, on the time scale of the reptation time $\tau_{w, L}$ of the long chains, the short molecules act as a solvent. The distance between entanglements of the long chains with each other and therefore the effective tube diameter of the "supertubes", representing the mean field of the interactions of the long chains among themselves, increases inversely proportional to the weight fraction $w$ of the long chains. In contrast to the dynamic dilution effect in linear viscoelasticity that leads to a reduction of the longest relaxation time $\tau_{w, L}$, the tube-diameter relaxation time $\tau_{D}$ of the diluted long chains, which represents a nonlinear viscoelastic property, is expected to increase in comparison to the tube-diameter relaxation time $\tau_{a, L}$ of a melt of the long molecules, according to a relation of the form

$$
\tau_{D}=\frac{\tau_{a, L}}{w^{\chi}}
$$

$\chi$ is a (positive) dilution exponent. $\tau_{D}$ represents the tube-diameter relaxation time of the supertube segments. As the tube diameter of a supertube segment is inversely proportional to the weight fraction $w$ of the long chains and its cross section is inversely proportional to the square of the weight fraction $w$, a dilution exponent $\chi=2$ may be expected, i.e., relaxation of the tube diameter of the supertube increases inversely proportional to the square of the weight fraction $w$ of the long-chain component. With $\tau_{D}$ [Eq. (15)] and restricting attention to the long chains, the evolution equation for $f^{2}$ can then be written as

$$
\frac{\partial f^{2}}{\partial t}=2 f^{2}\left[(\boldsymbol{\kappa}: S)-\frac{1}{\tau_{D}} f\left(f^{3}-1\right)\right],
$$

with the initial condition $f\left(t=t^{\prime}, t^{\prime}\right)=1$. Combining Eq. (16) with Eq. (2) using the relaxation spectra of the bidisperse blends as obtained from linear viscoelasticity (Table I), the strain hardening of the blends in the regime $D e_{S}<1$ can be predicted. We call this the "long-chain model" in the following, as the chain stretching of the long chains is also 


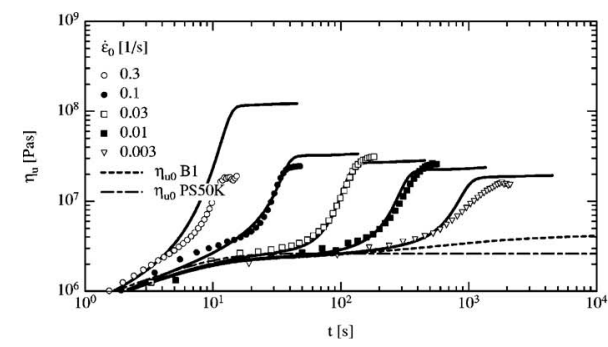

(a)

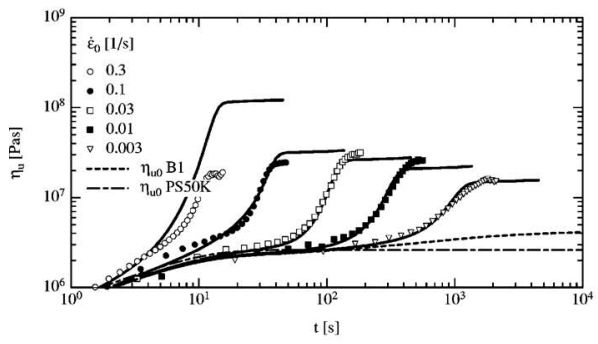

(b)

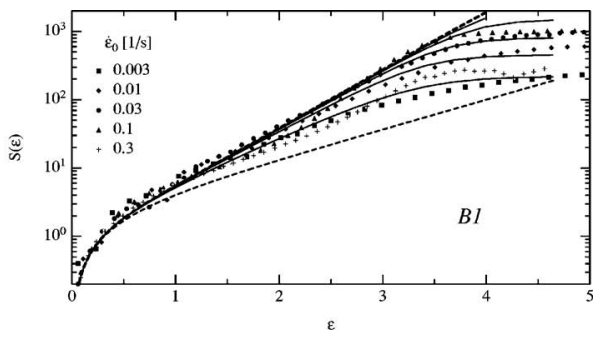

(c)

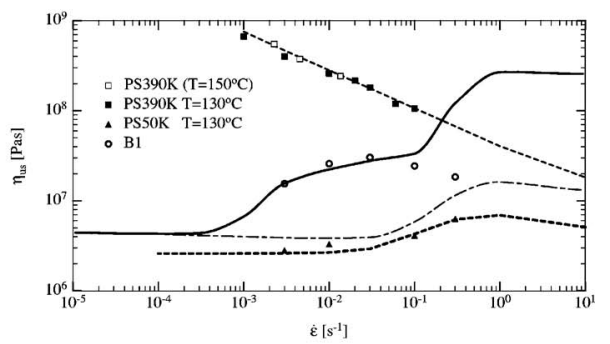

(d)

FIG. 1. (a) Comparison of measured transient elongational viscosity data (symbols) of Blend 1 at $130{ }^{\circ} \mathrm{C}$ to predictions (lines) of the long-chain model according to Eq. (16). (b) Comparison of measured transient elongational viscosity data (symbols) of Blend 1 at $130{ }^{\circ} \mathrm{C}$ to predictions (lines) of the long-chain model according to Eq. (18). (c) Strain measure data (symbols) of Blend 1 at $130^{\circ} \mathrm{C}$ compared to predictions of the long-chain model (lines) according to Eq. (18). Upper dotted line corresponds to the affine chain stretch hypothesis [Eq. (13)], and lower dotted line to the tube squeeze hypothesis [Eq. (10)]. (d) Comparison of measured steady-state elongational viscosity data (open dots) of Blend 1 at $130{ }^{\circ} \mathrm{C}$ to predictions of the long-chain model according to Eq. (18) with (full line) and without the effect of dilution (dash-dotted line).

assumed to be applicable to the short-chain component. Of course, as the short chains are expected to be stretched much less than the long chains, this model fails as soon as the nonlinear viscoelastic regime of the short chains is reached, i.e., for $D e_{S}>1$.

The time-dependent elongational viscosity data of Blend 1 (95.98\% PS50K and 4.02\% PS390K) are presented in Fig. 1(a). Also indicated are the linear-viscoelastic start-up viscosities of the blend and of the pure PS50K. With $\tau_{a, 390 \mathrm{~K}}=1462 \mathrm{~s}$ as observed by Wagner et al. (2005a) and a dilution exponent $\chi=2$, i.e.,

$$
\tau_{D}=\frac{1462}{w^{2}} \mathrm{~s}=9.05 \cdot 10^{5} \mathrm{~s}
$$

predictions of the MSF model [Eq. (2) in combination with Eq. (16)] are in reasonable agreement with experimental data for $\dot{\varepsilon} \leqslant 0.1 \mathrm{~s}^{-1}$.

It should be noted that agreement of the steady-state elongational viscosity measured and predicted essentially depends on the value of the tube-diameter relaxation time $\tau_{D}$ according to Eq. (17). Prediction of a steady-state elongational viscosity that is (at least initially) increasing with increasing strain rate is only possible if $\tau_{D}$ is larger than the longest relaxation time of the melt. This is due to the fact that while chain orientation depends on the Deborah number $D e_{O}=\dot{\varepsilon} \tau_{w}$, with $\tau_{w}$ being the longest relaxation time of the melt, chain stretch depends on the Deborah number $D e_{D}=\dot{\varepsilon} \tau_{D}$. If $D e_{O}>D e_{D}$, chain orientation occurs before chain stretch, and the steady-state elongational viscosity will decrease with increasing strain rate. On the other hand, if $D e_{O}<D e_{D}$, chain stretch will 
occur concurrently with chain orientation, and the steady-state elongational viscosity will increase with increasing strain rate. This is clearly the case for Blend 1 with $\tau_{D}$ $=9.05 \cdot 10^{5} \mathrm{~s}$, which is two orders of magnitude larger than $\tau_{w}=6.75 \cdot 10^{3} \mathrm{~s}$ (see Table I). The same is true in the case of pure PS50K leading to a pronounced maximum in the steady-state elongational viscosity as discussed by Wagner et al. (2005a). From Fig. 1(a), it is also obvious that the long-chain model fails as soon as stretching of the short chains starts, and the elongational viscosity becomes grossly overpredicted. We will return to this problem in connection with discussion of the Double Reptation model in Sec. V.

Good agreement of the slope measured and predicted is observed for $\dot{\varepsilon}$ equal to 0.1 and $0.03 \mathrm{~s}^{-1}$ in Fig. 1(a), while for the two smallest strain rates the measured increase in the transient elongational viscosity is increasingly smaller than predicted. Apparently, at small strain rates, chain stretch is no longer caused by affine chain stretch, according to Eq. (13), but an additional stretch relaxation process becomes important, effectively leading to a transition to stretch by tube squeeze as in the case of polydisperse linear polymer melts, according to Eq. (10). This crossover can be modeled by an evolution equation of the form

$$
\frac{\partial f^{2}}{\partial t}=2 f^{2}\left[(\boldsymbol{\kappa}: S)-\frac{w^{\chi}}{\tau_{a, L}} f\left(f^{3}-1\right)-\frac{1}{\tau_{s}} \frac{f-1}{f}\right],
$$

where $\tau_{s}$ is a stretch relaxation time. Prediction of the strain hardening slope at the two smallest elongation rates is remarkably improved by using Eq. (18) with $\tau_{s}=1000 \mathrm{~s}$ [Fig. $1(b)]$.

An alternative approach to analyze the experimental data is to convert the timedependent elongational viscosity data to the strain measure $S(\varepsilon, \dot{\varepsilon})$ by [Wagner $(1978)]$ :

$$
S(\varepsilon, \dot{\varepsilon})=\frac{\sigma(\varepsilon)}{G(\varepsilon)}-\frac{1}{\dot{\varepsilon}} \int_{0}^{\varepsilon} \sigma\left(\varepsilon^{\prime}\right) \frac{m\left(\varepsilon^{\prime}\right)}{G^{2}\left(\varepsilon^{\prime}\right)} d \varepsilon^{\prime}=5 f^{2}\left(S_{11}-S_{33}\right),
$$

where $\sigma$ is the elongational stress and $\varepsilon$ the Hencky strain. $m$ and $G$ are the memory function and the relaxation modulus, and $S_{11}$ and $S_{33}$ are the components of the orientation tensor in the stretching and the transversal direction, respectively. The second equality in Eq. (19) follows directly from Eqs. (2) and (5). The converted experimental data are presented in Fig. 1(c). The strain measure increases with increasing strain $\varepsilon$, reaching plateau values at high strains that increase at first with increasing strain rate $\dot{\varepsilon}$, before decreasing again for the highest measured strain rate. It is obvious that at higher strains, time-strain separability is not observed. Also shown in Fig. 1(e) are predictions of Eqs. (9) and (12) representing the strain measures resulting from (nonaffine) tube squeeze and affine chain stretch, respectively, in the hyperelastic limit. In the hyperelastic regime, i.e., before the experimentally determined strain measures bend over to the plateau values, the experimental data lie between the predictions of Eqs. (9) and (12), approaching at small strain rates the tube squeeze prediction of Eq. (9), while at higher strain rates they follow the affine stretch hypothesis as represented by Eq. (12). Predictions of the chain dilution approach as formulated by Eq. (18) are in reasonable agreement with experimental data except, as expected, for the highest elongation rate, where the long-chain model fails.

The steady-state elongational viscosity data of Blend 1 are presented in Fig. 1(d), together with the steady-state elongational viscosities of the blend components PS50K and PS390K. Prediction (full line) using the evolution equation [Eq. (18)] is in reasonable agreement with experimental data at strain rates $\dot{\varepsilon} \leqslant 0.1 \mathrm{~s}^{-1}$, while at higher strain rates, when stretching of the short chains starts, and the elongational viscosity is grossly overpredicted. Also shown is the predicted steady-state elongational viscosity of Blend 1 


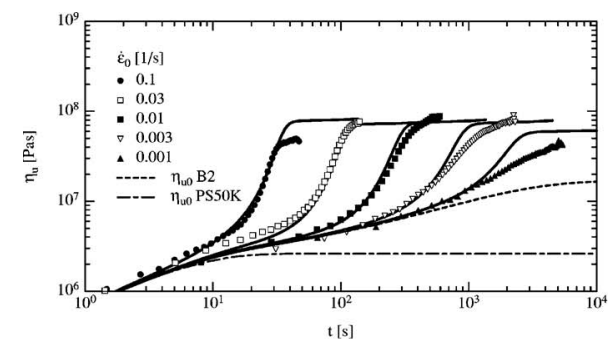

(a)

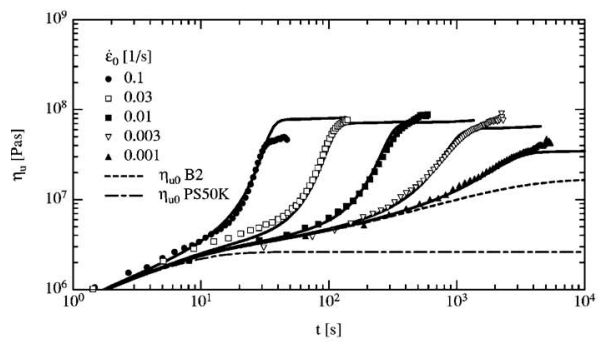

(b)

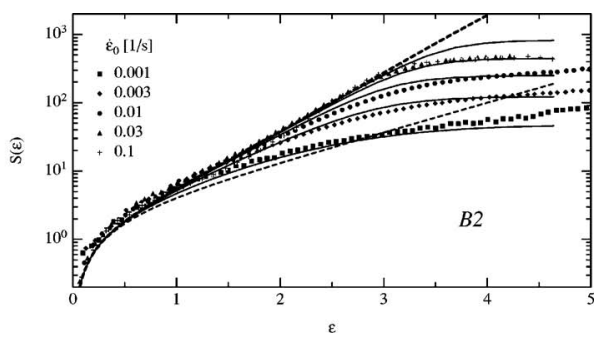

(c)

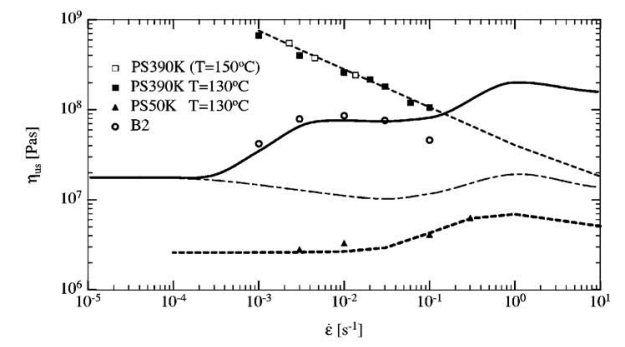

(d)

FIG. 2. (a) Comparison of measured transient elongational viscosity data (symbols) of Blend 2 at $130{ }^{\circ} \mathrm{C}$ to predictions (lines) of the long-chain model according to Eq. (16). (b) Comparison of measured transient elongational viscosity data (symbols) of Blend 2 at $130{ }^{\circ} \mathrm{C}$ to predictions (lines) of the long-chain model according to Eq. (18). (c) Strain measure data (symbols) of Blend 2 at $130^{\circ} \mathrm{C}$ compared to predictions of the long-chain model (lines) according to Eq. (18). Upper dotted line corresponds to the affine chain stretch hypothesis [Eq. (13)], and lower dotted line to the tube squeeze hypothesis [Eq. (10)]. (d) Comparison of measured steady-state elongational viscosity data (open dots) of Blend 2 at $130{ }^{\circ} \mathrm{C}$ to predictions of the long-chain model according to Eq. (18) with (full line) and without the effect of dilution (dash-dotted line).

assuming no dilution effect of the tube-diameter relaxation time, i.e., by setting $w=1$ in Eq. (18) [dash-dotted line in Fig. 1(d)]. Comparison of the two predictions clearly indicates the importance of the effect of dilution in understanding the elongational viscosity of the bidisperse blend.

To verify the results obtained for Blend 1, the same analysis is now applied to Blend 2 (85.63\% PS50K and $14.37 \%$ PS390K). In this case, a dilution exponent of $\chi=2.7$ was found, i.e.,

$$
\tau_{D}=\frac{1462}{w^{2.7}} \mathrm{~s}=2.75 \cdot 10^{5} \mathrm{~s}
$$

Thus, $\tau_{D}$ of Blend 2 is significantly smaller than $\tau_{D}$ of Blend 1, which is in general agreement with the dilution concept. The dilution exponent of Blend 2 is found to be larger than the dilution exponent of Blend 1. However, it should be kept in mind that due to its small molar mass, the matrix polymer PS50K possesses only a few (3-4) entanglements, and deviations from any ideal scaling are to be expected.

The time-dependent elongational viscosity data of Blend 2 are presented in Figs. 2(a) and 2(b) together with predictions of the MSF model. Again, using evolution equation [Eq. (16)] the slope of the transient elongational viscosity is overpredicted at small strain rates. This can be rectified by use of evolution equation [Eq. (18)] with a stretch relaxation time of $\tau_{s}=1000 \mathrm{~s}$, as shown in Fig. 2(b). The transition from affine chain stretch to tube squeeze is clearly seen in Fig. 2(c), where experimentally determined and predicted 


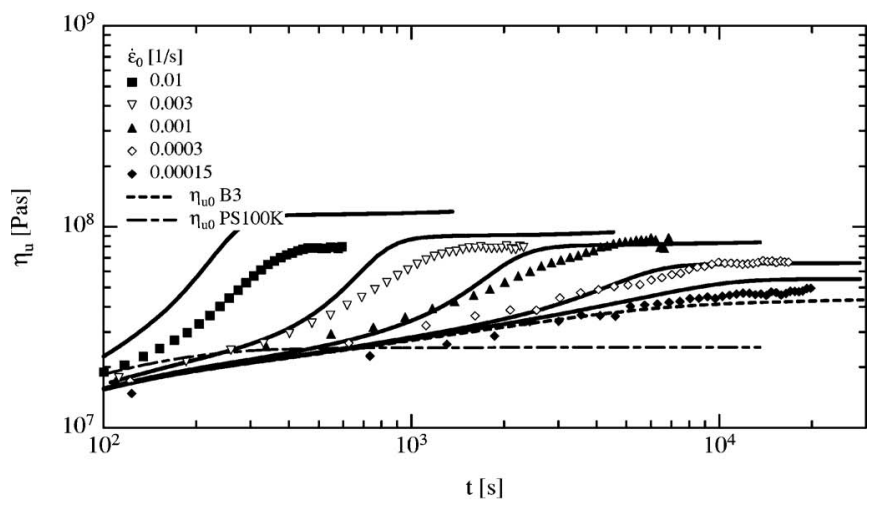

(a)

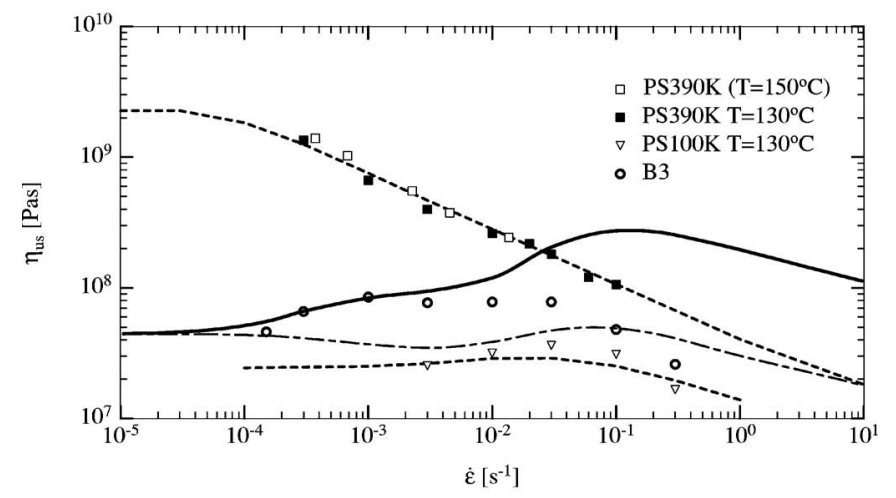

(b)

FIG. 3. (a) Comparison of measured transient elongational viscosity data (symbols) of Blend 3 at $130{ }^{\circ} \mathrm{C}$ for the five lowest strain rates measured to predictions (lines) of the long-chain model according to Eq. (16). (b) Comparison of measured steady-state elongational viscosity data (open dots) of Blend 3 at $130{ }^{\circ} \mathrm{C}$ to predictions of the long-chain model according to Eq. (16) with (full line) and without the effect of dilution (dashdotted line).

strain measures show good agreement, except for the highest strain rate. Again, the long-chain model fails as soon as stretching of the short chains begins, i.e., at $D e_{S}$ $=\dot{\varepsilon} \tau_{w, S}>1$, as seen in Fig. 2(d), where the steady-state elongational viscosity data are compared to predictions of the MSF model with evolution equation [Eq. (18)] (full line). Also given in Fig. 2(d) is the predicted steady-state elongational viscosity of Blend 2 assuming no dilution effect of the tube-diameter relaxation time, i.e., for $w=1$ in Eq. (18) [dash-dotted line in Fig. 2(d)]. Again, comparison of the two predictions clearly indicates the importance of the dilution effect in explaining the elongational viscosity of bidisperse blends.

For Blend $3(85.98 \%$ PS100K and $14.02 \%$ PS390K), the difference in the molar masses of the blend components is smaller and the molar mass of the short-chain matrix is higher than in the cases of Blend 1 and Blend 2. Therefore, the elongation-rate regime where the long-chain model is expected to hold is reduced and shifted to smaller strain rates. The time-dependent and steady-state elongational viscosity data of Blend 3 are presented in Fig. 3(a) (restricted to the five smallest strain rates measured) and Fig. 3(b), respectively. Also indicated are the linear-viscoelastic start-up viscosities of the blend and 
of the pure PS100K [Fig. 3(a)] as well as the steady-state elongational viscosities of the blend components PS100K and PS390K [Fig. 3(b)]. With $\tau_{a, 390 \mathrm{~K}}=1462 \mathrm{~s}$ and a dilution exponent $\chi=2$, i.e.,

$$
\tau_{D}=\frac{1462}{w^{2}} \mathrm{~s}=7.44 \cdot 10^{4} \mathrm{~s}
$$

predictions of the MSF model using the evolution equation [Eq. (16)] are in qualitative agreement with experimental data for $\dot{\varepsilon} \leqslant 0.003 \mathrm{~s}^{-1}$. Agreement could be improved by introducing a stretch relaxation time $\tau_{s}$, according to Eq. (18), but due to the restricted validity of the long-chain model in the case of Blend 3, the improvement is minor and therefore not shown here.

There are two factors contributing to the reduced amount of strain hardening observed for Blend 3 in comparison to Blend 1 and Blend 2: (1) a smaller chain stretch caused by a smaller tube-diameter relaxation time $\tau_{D}$ together with a shift of the dilution regime to smaller strain rates. In fact, the square of the maximum stretch, $f_{\max }^{2}$, is given by:

$$
f_{\max }^{2} \approx \sqrt{D e_{D}}=\sqrt{\dot{\varepsilon} \tau_{D}} .
$$

As both $\tau_{D}$ and $\dot{\varepsilon}$ are smaller, the maximum stretch is reduced. (2) The steady-state elongational viscosity of the matrix component PS100K is much higher than in the case of PS50K, and therefore the viscosity of the "solvent" component is masking the contribution of the long-chain component. Even so, the dilution effect on the nonlinear viscoelasticity of Blend 3 is again demonstrated in Fig. 3(b) by comparison of predictions with and without the effect of dilution on $\tau_{D}$. At $D e_{S}=\dot{\varepsilon} \tau_{w, S}>1$, when stretching of the short chains starts, the long-chain model fails and the elongational viscosities are overpredicted.

\section{A NONLINEAR EXTENSION OF THE DOUBLE REPTATION MODEL}

Until this stage, the analysis was restricted to the terminal zone of the relaxation spectrum of the blends, which is dominated by the long chains. A full model has to take into account the interactions of long and short molecules, and their effect on the relaxation spectrum, as well as the difference in stretching of long and short chains. In recent years, several elaborate models have been developed to relate molar mass distribution and linear viscoelasticity of polymer melts [see, e.g., Tuminello (1986)]. The extension of these models to nonlinear viscoelasticity is beyond the scope of this article. We will restrict ourselves to the basic Double Reptation Model, which in the bidisperse case reduces to a quadratic mixing rule of the form [Tsenoglou (1987); des Cloizeaux (1998, 1990); Tsenoglou (1991); Wasserman and Graessley (1992)]

$$
\stackrel{\circ}{G}(t)=\left[w \sqrt{g_{L}(t)}+(1-w) \sqrt{g_{S}(t)}\right]^{2} .
$$

$\stackrel{\circ}{G}(t)$ is the linear-viscoelastic relaxation modulus of the blend, and $g_{L}(t)$ and $g_{S}(t)$ are the linear-viscoelastic relaxation moduli of the long (L) and short chain $(\mathrm{S})$ blend components, respectively, which are taken from Wagner et al. (2005a), and are presented in the form of discrete relaxation spectra for easy reference in Table II. From Eq. (23), the memory function $m\left(t-t^{\prime}\right)$ is obtained as 
TABLE III. Empirical shift factors to account for dynamic tube dilution.

\begin{tabular}{|c|c|c|c|c|c|c|}
\hline \multirow[b]{2}{*}{$\begin{array}{l}\text { Shift } \\
\text { Factors }\end{array}$} & \multicolumn{2}{|c|}{ PS50K } & \multicolumn{2}{|c|}{ PS100K } & \multicolumn{2}{|c|}{ PS390K } \\
\hline & $g_{i}[\mathrm{~Pa}]$ & $\lambda_{i}[\mathrm{~s}]$ & $g_{i}[\mathrm{~Pa}]$ & $\lambda_{i}[\mathrm{~s}]$ & $g_{i}[\mathrm{~Pa}]$ & $\lambda_{i}[\mathrm{~s}]$ \\
\hline B1 & 1 & 0.6 & & & 8 & 0.06 \\
\hline B2 & 1 & 0.6 & & & 2.8 & 0.1 \\
\hline B3 & & & 1 & 0.5 & 1.7 & 0.3 \\
\hline
\end{tabular}

$$
m\left(t-t^{\prime}\right)=-\frac{d \stackrel{\circ}{G}\left(t-t^{\prime}\right)}{d t}=\sqrt{\stackrel{\circ}{G}\left(t-t^{\prime}\right)}\left[w \frac{m_{L}\left(t-t^{\prime}\right)}{\sqrt{g_{L}\left(t-t^{\prime}\right)}}+(1-w) \frac{m_{S}\left(t-t^{\prime}\right)}{\sqrt{g_{S}\left(t-t^{\prime}\right)}}\right],
$$

with the memory functions $m_{L}\left(t-t^{\prime}\right)$ and $m_{S}\left(t-t^{\prime}\right)$ of the long- and short-chain components, respectively.

Unfortunately, this simple quadratic mixing rule does not allow a quantitative description of the linear viscoelasticity of the blends. The reason is that when the difference in molar masses of the blend components is large, dynamic tube dilation for the long-chain component already takes place in the linear-viscoelastic regime, reducing the reptation time of the long chains [Doi et al. (1987)]. To have a guideline when this linearviscoelastic tube dilation process should be taken into account, Struglinski and Graessley (1985) defined the parameter:

$$
G_{r}=\frac{M_{L} M_{e}^{2}}{M_{S}^{3}}
$$

$M_{e}$ is the molar mass between entanglements and is taken as $13.3 \mathrm{~kg} \mathrm{~mol}^{-1}$ here [Fetters et al. (1994)]. The tube dilation effect is considered relevant as soon as $G_{r}>0.064$ [Park and Larson (2004)], a condition that is fulfilled by all three of the bidisperse blends analysed here (see Table I), although for Blend 3 only marginally so. We can account for this linear-viscoelastic tube dilution effect empirically by shifting the spectra of the short-chain and long-chain blend components by shift factors in the horizontal or time direction, and in the vertical or modulus direction until reasonable agreement between measured and predicted zero shear-rate viscosities is achieved. The shift factors used in the following are summarized in Table III. The smallest shifts were needed for Blend 3. Therefore we consider Blend 3 first.

We now propose a nonlinear viscoelastic extension of Eq. (23) by

$$
G(t)=\left[w \sqrt{g_{L}(t)} f_{L}+(1-w) \sqrt{g_{S}(t)} f_{S}\right]^{2} .
$$

The molecular stress functions $f_{L}$ and $f_{S}$ are those of the long-chain and short-chain components. The corresponding single integral constitutive equation then takes the form

$$
\begin{aligned}
\boldsymbol{\sigma}(t)= & w^{2} \int_{-\infty}^{t} m_{L} f_{L}^{2} S_{D E}^{I A} d t^{\prime}+w(1-w) \int_{-\infty}^{t}\left[\sqrt{\frac{g_{L}}{g_{S}}} m_{S}+\sqrt{\frac{g_{S}}{g_{L}}} m_{L}\right] f_{L} f_{S} S_{D E}^{I A} d t^{\prime} \\
& +(1-w)^{2} \int_{-\infty}^{t} m_{S} f_{S}^{2} S_{D E}^{I A} d t^{\prime} .
\end{aligned}
$$

The time dependences are dropped for simplicity. The stress $\boldsymbol{\sigma}$ is seen to be the sum of three terms, the contribution of the long and short chains with quadratic weight, and a mixed term representing the interaction of short and long chains. 


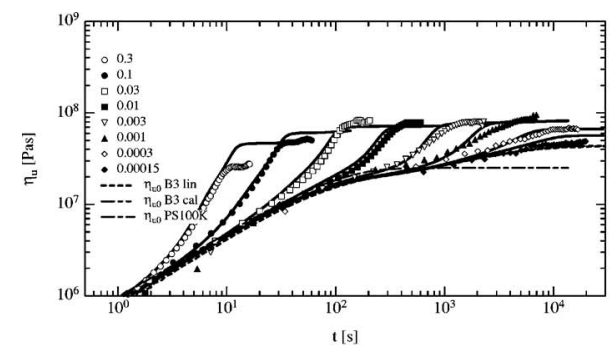

(a)

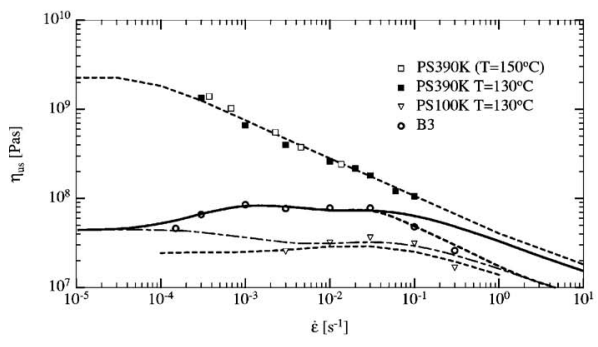

(b)

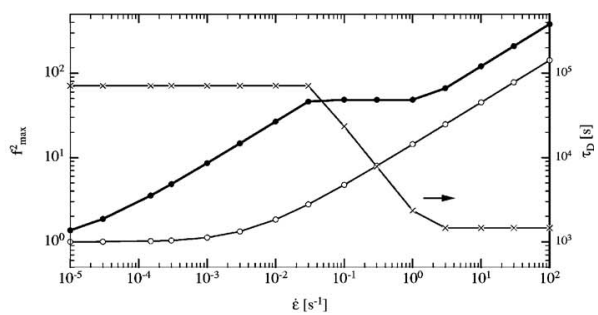

(c)

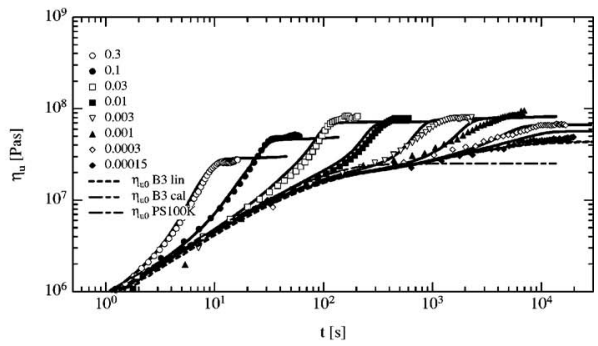

(d)

FIG. 4. (a) Comparison of measured transient elongational viscosity data (symbols) of Blend 3 at $130{ }^{\circ} \mathrm{C}$ to predictions (lines) of the Double Reptation Model according to Eq. (27)-Eq. (29). (b) Comparison of measured steady-state elongational viscosity data (open dots) of Blend 3 at $130{ }^{\circ} \mathrm{C}$ to prediction of the Double Reptation Model according to Eq. (27)-Eq. (29) with (full line) and without the effect of dilution (dash-dotted line). The transition from the dilution to the undiluted regime, according to Eq. (31), is indicated (heavy dotted line). (c) $f_{\max }^{2}$ of long-chain (full dots) and short-chain (open dots) component of Blend 3 as a function of elongation rate. $\tau_{D}$ is the tube-diameter relaxation time of the long-chain component, according to Eq. (31). (d) Comparison of measured transient elongational viscosity data (symbols) of Blend 3 at $130^{\circ} \mathrm{C}$ to predictions (lines) of the Double Reptation Model, according to Eq. (27)-Eq. (29) and Eq. (31).

For the molecular stress functions $f_{S}$ and $f_{L}$, the following evolution equations are used:

For $f_{S}$ :

$$
\frac{\partial f_{S}^{2}}{\partial t}=2 f_{S}^{2}\left[(\boldsymbol{\kappa}: S)-\frac{1}{\tau_{a, S}} f_{S}\left(f_{S}^{3}-1\right)\right],
$$

corresponding to Eq. (14).

For $f_{L}$ and Blend 3:

$$
\frac{\partial f_{L}^{2}}{\partial t}=2 f_{L}^{2}\left[(\boldsymbol{\kappa}: S)-\frac{1}{\tau_{D}} f_{L}\left(f_{L}^{3}-1\right)\right],
$$

corresponding to Eq. (16), and for Blends 1 and 2:

$$
\frac{\partial f_{L}^{2}}{\partial t}=2 f_{L}^{2}\left[(\boldsymbol{\kappa}: S)-\frac{1}{\tau_{D}} f_{L}\left(f_{L}^{3}-1\right)-\frac{1}{\tau_{s}} \frac{f_{L}-1}{f_{L}}\right],
$$

corresponding to Eq. (18).

Figures 4(a) and 4(b) present the transient and steady-state elongational viscosities measured for Blend 3 as well as predictions of the nonlinear Double Reptation Model using Eq. (27) to Eq. (29) together with the parameters $\tau_{D}$ according to Eq. (21), and $\tau_{a, S}=\tau_{a, 100 \mathrm{~K}}=203 \mathrm{~s}$. It is clearly seen that the model in its present form gives a reason- 
able description of the elongational viscosities at small and intermediate strain rates, while it fails for the two highest strain rates. This is due to the assumption of a continuous increase in stretching of the long chain component as prescribed by Eq. (29). In contrast, it is obvious from Fig. 4(b) that the steady-state elongational viscosity measured at the highest elongation rate of $\dot{\varepsilon}=0.3 \mathrm{~s}^{-1}$ is comparable to a viscosity predicted without taking into account the effect of dilution, i.e. by use of Eq. (21) with $w=1$ [dash-dotted line in Fig. 4(b)]. Thus, it appears that there exists a transition from the dilution regime to a regime where the tube-diameter relaxation time of the long-chain component corresponds to the tube-diameter relaxation time of the bulk. To account for this effect, we propose the following hypothesis: The dilution regime ends as soon the tube diameter $a_{L}$ of the supertube created by the interaction of the long molecules among themselves, has been reduced by deformation from its equilibrium value $a_{L 0}$ to the tube diameter $a_{0}$ of the bulk polymer. The critical maximum stretch $f_{L c}$ of the long-chain component which defines the end of the full dilution regime is then given by:

$$
f_{L c}^{2}=\frac{a_{L 0}^{2}}{a_{L}^{2}}=\frac{a_{L 0}^{2}}{a_{0}^{2}}=\frac{a_{0}^{2} / w^{\chi}}{a_{0}^{2}}=\frac{1}{w^{\chi}} .
$$

We thereby assume that the dilution exponents of the supertube cross section $a_{L 0}^{2}$ $=a_{0}^{2} / w^{\chi}$, and of the tube-diameter relaxation time are the same. It then follows from Eqs. (22) and (30) that the tube-diameter relaxation time is not a constant anymore, but a function of the elongation rate applied. As soon as the full dilution regime ends, the tube-diameter relaxation time $\tau_{D}$ of the long chains decreases from the fully "diluted" value of $\tau_{D}=\tau_{a, L} / w^{\chi}$ to the bulk value of $\tau_{D}=\tau_{a, L}$ in such a way that the maximum stretch of the long chains remains constant at $f_{L \max }^{2}=f_{L c}^{2}$. For constant strain-rate elongational flow, this can be expressed by the equations:

$$
\begin{gathered}
\tau_{D}=\frac{\tau_{a, L}}{w^{\chi}} \quad \text { for } \dot{\varepsilon} \leqslant \frac{1}{\tau_{a, L^{w^{\chi}}}}=\dot{\varepsilon}_{c}, \\
\tau_{D}=\frac{1}{w^{2 \chi} \dot{\varepsilon}} \quad \text { for } \dot{\varepsilon}_{c} \leqslant \dot{\varepsilon} \leqslant \frac{\dot{\varepsilon}_{c}}{w^{\chi}}, \\
\tau_{D}=\tau_{a, L} \quad \text { for } \dot{\varepsilon} \geqslant \frac{\dot{\varepsilon}_{c}}{w^{\chi}} .
\end{gathered}
$$

$\dot{\varepsilon}_{c}$ is the critical strain rate, at which $f_{L c}$ is reached. Equations (31), together with predictions of Eqs. (28) and (29), are presented in Fig. 4(c) for Blend 3 with $\chi=2 . \tau_{D}$ remains constant until the critical strain rate $\dot{\varepsilon}_{c}$ is reached, and then decreases inverse proportional to $\dot{\varepsilon}$ to a value of $\tau_{D}=\tau_{a, L}$. During the decrease of $\tau_{D}$, the maximum stretch of the long chains remains constant at $f_{L \text { max }}^{2}=f_{L c}^{2}$. At still higher strain rates, the stretching of the long-chain component is expected to show the same behavior as in a melt of long chains, i.e., increasing proportionally to the square root of the strain rate as prescribed by Eq. (22). Figures 4(b) and 4(d) show that indeed, the high strain-rate predictions of the Double Reptation Model for both the steady-state and the transient elongational viscosities are greatly improved.

Of course, instead of the Eqs. (31), a generalized and deformation invariant crossover function from the dilution regime [Eq. (31a)] to the bulk regime [Eq. (31c)] could be proposed. However, in view of the limited data basis, we refrain from doing so here.

Using the same approach, predictions of the nonlinear Double Reptation Model for Blend 2 (Fig. 5) and Blend 1 (Fig. 6) with a tube diameter relaxation time for the matrix 


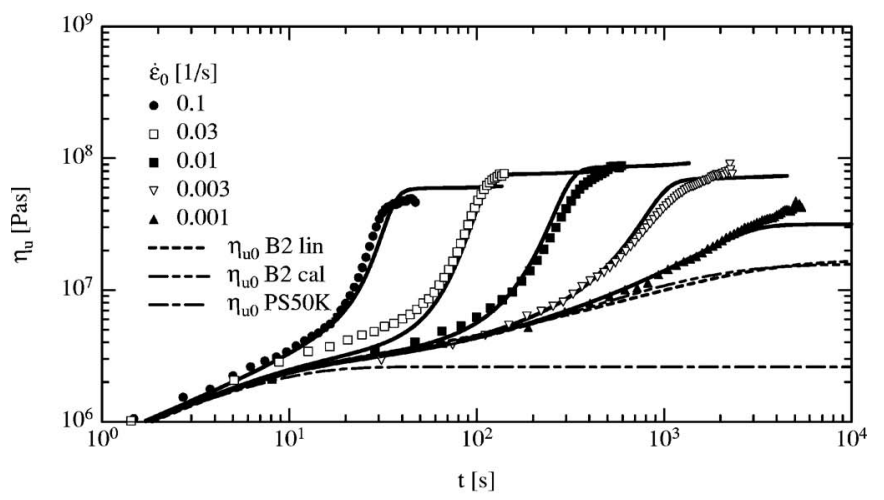

(a)

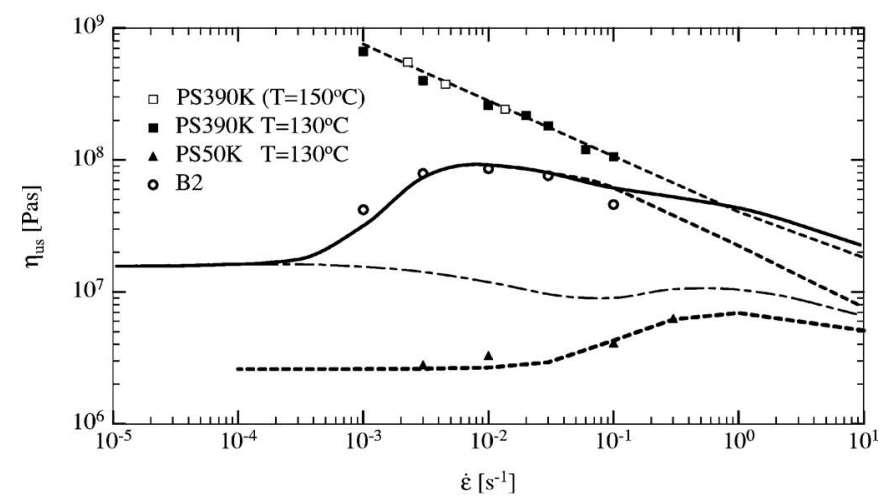

(b)

FIG. 5. (a) Comparison of measured transient elongational viscosity data (symbols) of Blend 2 at $130{ }^{\circ} \mathrm{C}$ to predictions (lines) of the Double Reptation Model, according to Eq. (27)-Eq. (29) and Eq. (31). (b) Comparison of measured steady-state elongational viscosity data (open dots) of Blend 2 at $130{ }^{\circ} \mathrm{C}$ to prediction of the Double Reptation Model, according to Eq. (27)-Eq. (29) with (full line) and without the effect of dilution (dash-dotted line). The transition from the dilution to the undiluted regime, according to Eq. (31), is indicated (heavy dotted line).

PS50K, $\tau_{a, S}=\tau_{a, 50 \mathrm{~K}}=141 \mathrm{~s}$, a stretch relaxation time $\tau_{s}=1000 \mathrm{~s}$, and a dilution exponent of $\chi=2.7$ (Blend 2) and $\chi=2$ (Blend 1) are seen to be in general agreement with experimental evidence. Again, it seems reasonable to assume that the tube-diameter relaxation time of the long-chain component returns to its value in the undiluted bulk, and consequently, at higher elongation rates, the steady-state elongational viscosity approaches the values predicted without the dilution effect, although the experimental evidence is more restricted than in the case of Blend 3.

\section{CONCLUSIONS}

In this contribution we have analyzed the transient and steady-state elongational viscosity data of three bidisperse polystyrene blends investigated recently by Nielsen et al. (2006). The framework applied for the constitutive analysis is the MSF model of Wagner et al. (2005a), which is based on the assumption of a strain-dependent tube diameter and the interchain pressure term of Marrucci and Ianniruberto (2004). 


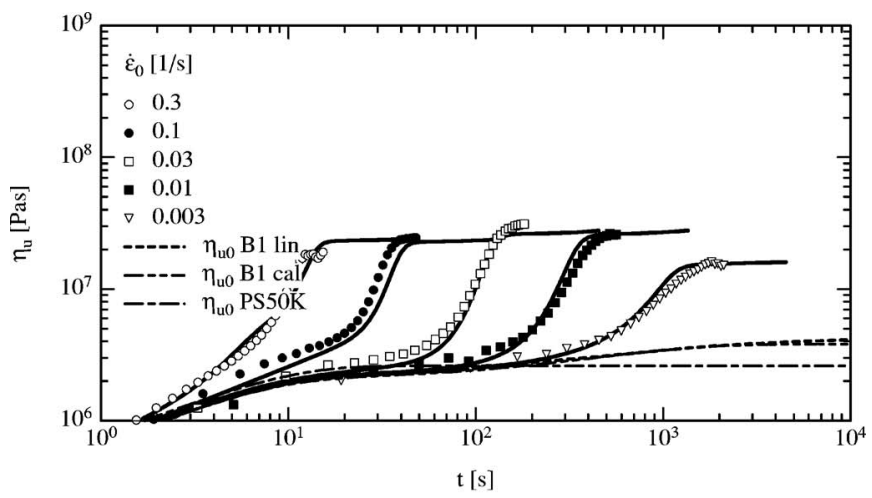

(a)

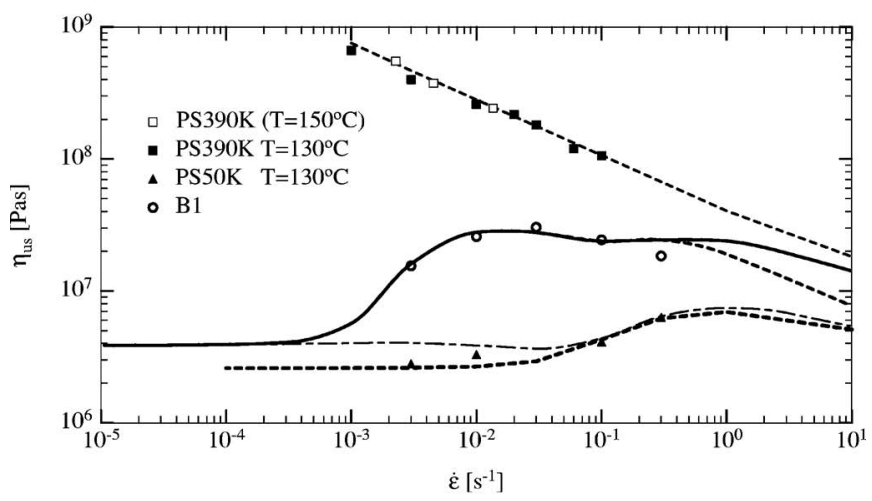

(b)

FIG. 6. (a) Comparison of measured transient elongational viscosity data (symbols) of Blend 1 at $130{ }^{\circ} \mathrm{C}$ to predictions (lines) of the Double Reptation Model, according to Eq. (27)-Eq. (29) and Eq. (31). (b) Comparison of measured steady-state elongational viscosity data (open dots) of Blend 1 at $130{ }^{\circ} \mathrm{C}$ to prediction of the Double Reptation Model, according to Eq. (27)-Eq. (29) with (full line) and without the effect of dilution (dash-dotted line). The transition from the dilution to the undiluted regime, according to Eq. (31), is indicated (heavy dotted line).

In analyzing the strain-hardening behavior of the bidisperse blends with the long chains representing the minority component with a weight fraction $w \ll 1$, several distinctive phenomena can be identified in different strain-rate regimes. For elongational flows with Deborah numbers $D e_{S}=\dot{\varepsilon} \tau_{w, S}<1$, the short chain matrix represents a solvent. Interactions of dilute long chains among themselves create supertubes with enormously large tube-diameter relaxation times $\tau_{D}$, which are larger by a factor $1 / w^{\chi}$ than the tubediameter relaxation time $\tau_{a, L}$ of a melt of long chains. For the dilution exponent $\chi$, values of 2 and 2.7 were found. Considering the fact that the matrix polymers PS50K and PS100K have only few entanglements, it is not surprising that no unique scaling behavior is found. A large tube-diameter relaxation time $\tau_{D}$ leads to a large stretching potential of the long-chain component, and to an increase of the steady-state elongational viscosity with increasing strain rate. In addition, in this dilution regime, a transition from affine chain stretch to nonaffine tube squeeze with decreasing strain rate is caused by an additional stretch relaxation process.

As soon as the tube diameter $a_{L}$ of the supertube created by the interaction of the long molecules among themselves has been reduced by deformation from its equilibrium value 
$a_{L 0}$ to the tube diameter $a_{0}$ of the bulk polymer, the dilution regime ends. We propose the hypothesis that when the dilution regime ends, the tube-diameter relaxation time $\tau_{D}$ of the long-chain component decreases from the fully diluted value of $\tau_{D}=\tau_{a, L} / w^{\chi}$ to the bulk value of $\tau_{D}=\tau_{a, L}$ in such a way that the maximum stretch of the long chains remains effectively constant. A nonlinear extension of the basic double reptation concept was developed, which allows (albeit by use of empirical linear-viscoelastic shift factors to correct the linear-viscoelastic predictions) to obtain a quantitative description of the transient and steady-state elongational viscosities comprising all phenomena discussed previously.

Considering the implications of these findings for the understanding of the strainhardening behavior of polydisperse linear melts, we may make three statements. (1) Whenever a maximum in the steady-state elongational viscosity is observed this is most likely due to the effect of diluted long chains. (2) The effect is expected to be larger the higher the molar mass and the smaller the mass fraction of this component, provided that the mass fraction is high enough so that the long chains remain entangled with each other. (3) The origin of a constant maximum stretch $f_{\max }$, which has been observed in many polydisperse linear melts for a wide range of strain rates [see, e.g., Wagner et al., 2001], may be related to the end of the dilution regime, when $\tau_{D}$ decreases inversely proportional to the strain rate, leading to a constant maximum stretch $f_{\max }$.

\section{ACKNOWLEDGMENTS}

V.H.R.-G. wishes to thank the Consejo Nacional de Ciencia y Tecnología and Deutscher Akademischer Austauschdienst (CONACyT-DAAD) for the financial support. Financial support of the German Research Foundation (DFG) for part of this work is gratefully acknowledged.

\section{References}

Bach, A., K. Almdal, H. K. Rasmussen, and O. Hassager, "Elongational viscosity of narrow molar mass distribution polystyrene," Macromolecules 36, 5174-5179 (2003a).

Bach, A., H. K. Rasmussen, and O. Hassager, "Extensional viscosity for polymer melts measured in the filament stretching rheometer," J. Rheol. 47, 429-441 (2003b).

Berry, G. C., and T. G. Fox, "The viscosity of polymers and their concentrated solutions," Adv. Polym. Sci. 5, 261-357 (1968).

Daoud, M., and P. G. de Gennes, "Some remarks on the dynamics of polymer melts," J. Polym. Sci., Polym. Phys. Ed. 17, 1971-1981 (1979).

de Gennes, P. G., "Reptation of a polymer chain in the presence of fixed obstacles," J. Chem. Phys. 55, 572-579 (1971).

des Cloizeaux, J., "Double reptation vs. simple reptation in polymer melts," Europhys. Lett. 5, 437-442 (1988).

des Cloizeaux, J., "Relaxation and viscosity anomaly of melts made of long entangled polymers, timedependent reptation," Macromolecules 23, 4678-4687 (1990).

Doi, M., and S. F. Edwards, "Dynamics of Concentrated Polymer Systems. Part 2.- Molecular Motion under Flow,” J. Chem. Soc., Faraday Trans. 74, 1802-1817 (1978).

Doi, M., and S. F. Edwards, "Dynamics of Concentrated Polymer Systems. Part 4.- Rheological Properties," J. Chem. Soc., Faraday Trans. 75, 38-54 (1979).

Doi, M., "Explanation for the 3.4 power law of viscosity of polymeric liquids on the basis of the tube model," Polym. Lett. 19, 265-273 (1981).

Doi, M., "Explanation for the 3.4-power law for viscosity of polymeric liquids on the basis of the tube model," J. Polym. Sci., Polym. Phys. Ed. 21, 667-684 (1983). 
Doi, M., and S. F. Edwards, The Theory of Polymer Dynamics (Oxford University Press, Oxford, 1986).

Doi, M., W. W. Graessley, E. Helfand, and D. S. Pearson, "Dynamics of polymer in polydisperse melts," Macromolecules 20, 1900-1906 (1987).

Fetters, L. J., D. J. Lohse, D. Richter, T. A. Witten, and A. Zirkel, "Connection between polymer molecular weight, density, chain dimensions, and melt viscoelastic properties," Macromolecules 27, 4639-4647 (1994).

Frischknecht, A. L., and S. T. Milner, "Linear rheology of binary melts from a phenomenological tube model of entangled polymers," J. Rheol. 46, 671-684 (2002).

Gupta, R. K., D. A. Nguyen, and T. Sridhar, "Extensional viscosity of dilute polystyrene solutions: Effect of concentration and molecular weight," Phys. Fluids 12, 1296-1318 (2000).

Hua, Ch. C., and J. D. Schieber, "Segment connectivity, chain-length breathing, segmental stretch, and constraint release in reptation models. I. Theory and single-step strain predictions," J. Chem. Phys. 109, 10018-10027 (1998).

Isono, Y., K. Itoh, T. Komiyatani, and T. Fujimoto, "Differential dynamic modulus of polyisobutylene with high molecular weight 1, single-step large shearing deformations," Macromolecules 24, 4429-4432 (1991).

Ketzmerick, R., and H. C. Öttinger, "Simulation of a Non-Markovian process modelling contour length fluctuation in the Doi-Edwards model," Continuum Mech. Thermodyn. 1, 133-124 (1989).

Larson, R. G., Constitutive Equations for Polymer Melts and Solutions (Buttherworths, USA, 1988).

Luap, C., Ch. Müller, T. Schweizer, and D. C. Venerus, "Simultaneous stress and birefringence measurements during uniaxial elongation of polystyrene melts with narrow molecular weight distribution," Rheol. Acta 45, 83-91 (2005).

Marrucci, G., and J. J. Hermans, "Nonlinear viscoelasticity of concentrated polymer liquids," Macromolecules 13, 380-387 (1980).

Marrucci, G., “A model for polydisperse polymers," J. Polym. Sci., Polym. Phys. Ed. 23, 159-177 (1985).

Marrucci, G., and N. Grizzuti, "Fast flows of concentrated polymers: Predictions of the tube model on chain stretching," Gazz. Chim. Ital. 118, 179-185 (1988).

Marrucci, G., and G. Ianniruberto, "Interchain pressure effect in extensional flows of entangled polymer melts," Macromolecules 37, 3934-3942 (2004).

Masuda, T., K. Kitagawa, Z. Inoue, and S. Onogi, "Rheological properties of anionic polystyrenes II. Dynamic viscoelasticity of blends of narrow-distribution polystyrenes," Macromolecules 3, 116-125 (1970).

Mead, D. W., and L. G. Leal, "The reptation model with segmental stretch. I. Basic equations and general properties," Rheol. Acta 34, 339-359 (1995).

Milner, S. T., and T. C. B. McLeish, "Reptation and contour-length fluctuations in melts of linear polymers," Phys. Rev. Lett. 81, 725-728 (1998).

Nielsen, J. K., H. K. Rasmussen, O. Hassager, and G. H. McKinley, "Elongational viscosity of monodisperse and bidisperse polystyrene melts," J. Rheol. 50, 453-476 (2006).

O'Connor, N. P. T., and R. C. Ball, “Confirmation of the Doi-Edwards model,” Macromolecules 25, 5677-5682 (1992).

Öttinger, H. C., "A thermodynamically admissible reptation model for fast flows of entangled polymers," J. Rheol. 43, 1461-1493 (1999).

Park, S. J., and Larson, R. G., "Tube dilation and reptation in binary blends of monodisperse linear polymers," Macromolecules 37, 597-604 (2004).

Rolón-Garrido, V. H., M. H. Wagner, C. Luap, and T. Schweizer, "Modeling non-Gaussian extensibility effects in elongation of nearly monodisperse polystyrene melts," J. Rheol. 50, 327-340 (2006).

Struglinski, M. J., and W. W. Graessley, "Effects of polydispersity on the linear viscoelastic properties of entangled polymers. 1. Experimental observations for binary mixtures of linear polybutadiene," Macromolecules 18, 2630-2643 (1985).

Tsenoglou, C., "Viscoelasticity of binary homopolymer blends," Polym. Prepr. (Am. Chem. Soc. Div. Polym. Chem.) 28, 185-186 (1987).

Tsenoglou, C., "Molecular weight polydisperse effects on the viscoelasticity of entangled linear polymers," Macromolecules 24, 1762-1767 (1991).

Tuminello, W. H., "Molecular weight and molecular weight distribution from dynamic measurements of poly- 
mer melts," Polym. Eng. Sci. 26, 1339-1347 (1986).

Urakawa, O., M. Takahashi, T. Masuda, and N. G. Ebrahimi, "Damping functions and chain relaxation in uniaxial and biaxial extensions: Comparison with the Doi-Edwards theory," Macromolecules 28, 71967201 (1995).

van Ruymbeke, E., R. Keunings, and C. Bailly, "Determination of the molecular weight distribution of entangled linear polymers from linear viscoelasticity data," J. Non-Newtonian Fluid Mech. 105, 153-175 (2002).

Wagner, M. H., "A constitutive analysis of uniaxial elongational flow data of a low-density polyethylene melt," J. Non-Newtonian Fluid Mech. 4, 39-55 (1978).

Wagner, M. H., "The nonlinear strain measure of polyisobutylene melt in general biaxial flow and its comparison to the Doi-Edwards model," Rheol. Acta 29, 594-603 (1990).

Wagner, M. H., and J. Schaeffer, "Nonlinear measures for general biaxial extension of polymer melts," J. Rheol. 36, 1-26 (1992).

Wagner, M. H., H. Bastian, P. Hachmann, J. Meissner, S. Kurzbeck, H. Münstedt, and F. Langouche, "The strain-hardening behaviour of linear and long-chain-branched polyolefin melts in extensional flows," Rheol. Acta 39, 97-109 (2000).

Wagner, M. H., P. Rubio, and H. Bastian, "The molecular stress function model for polydisperse polymer melts with dissipative convective constraint release," J. Rheol. 45, 1387-1412 (2001).

Wagner, M. H., M. Yamaguchi, and M. Takahashi, "Quantitative assessment of strain hardening of low-density polyethylene melts by the molecular stress function model," J. Rheol. 47, 779-793 (2003).

Wagner, M. H., S. Kheirandish, and O. Hassager, "Quantitative prediction of transient and steady-state elongational viscosity of nearly monodisperse polystyrene melts," J. Rheol. 49, 1317-1327 (2005a).

Wagner, M. H., S. Kheirandish, and M. Yamaguchi, "Quantitative analysis of melt elongational behavior of LLDPE/LDPE blends," Rheol. Acta 44, 198-218 (2005b).

Wang, S., S. Q. Wang, A. Halasa, and W. L. Hsu, "Relaxation dynamics in mixtures of long and short chains: Tube dilation and impeded curvilinear diffusion," Macromolecules 36, 5355-5371 (2003).

Wasserman, S. H., and W. W. Graessley, "Effects of polydispersity on linear viscoelasticity in entangled polymer melts," J. Rheol. 36, 543-572 (1992).

Watanabe, H., T. Sakamoto, and T. Kotaka, "Entanglements in linear polystyrenes," Macromolecules 18, 1436-1442 (1985).

Winter, H. H., and M. Mours, IRIS Developments, 〈http://rheology.tripod.com/〉 (2003).

Ye, X., R. G. Larson, C. Pattamaprom, and T. Sridhar, "Extensional properties of monodisperse and bidisperse polystyrene solutions," J. Rheol. 47, 443-468 (2003).

Ylitalo, C. M., J. A. Kornfield, G. G. Fuller, and D. S. Pearson, "Molecular weight dependence of component dynamics in bidisperse melt rheology," Macromolecules 24, 749-758 (1991). 